\title{
On automorphisms of Enriques surfaces
}

\section{Dolgachev}

Max Planck Institut für Mathematik, Gottfried-Claren Straße 26, 5300 Bonn 3

Department of Mathematics, University of Michigan, Ann Arbor, Mi 48109, USA

To my teacher Igor Rostislavovich Shafarevich on the occasion of his sixtieth birthday

\section{Introduction}

An Enriques surface over an algebraically closed field $k$ of characteristic $\neq 2$ is a nonsingular projective surface $F$ with $H^{1}\left(F, \mathcal{O}_{F}\right)=H^{2}\left(F, \mathcal{O}_{F}\right)=0,2 K_{F}=0$. The unramified double cover of $F$ defined by the torsion class $K_{F}$ is a $\mathrm{K} 3$-surface $\bar{F}$, a nonsingular projective surface with $H^{1}\left(\bar{F}, \mathcal{O}_{F}\right)=0, K_{F}=0$. The study of Enriques surfaces is equivalent to the study of $\mathrm{K} 3$-surfaces with a fixed-point-free involution $\tau$. In particular, the automorphism group $\operatorname{Aut}(F)$ of $F$ is isomorphic to the group $\operatorname{Aut}(\bar{F}, \tau) /(\tau)$, where $\operatorname{Aut}(\bar{F}, \tau)$ is the centralizer of $\tau$ in the automorphism group $\operatorname{Aut}(\bar{F})$ of $\bar{F}$. In the case $k=\mathbb{C}$, the field of complex numbers, the study of $\operatorname{Aut}(\bar{F})$ is based on the Global Torelli Theorem for K3-surfaces proven by I. Piatetski-Shapiro and I. Shafarevich in [19]. It follows from this theorem that up to a finite group the group $\operatorname{Aut}(\bar{F})$ is isomorphic to the quotient group $O(\operatorname{Pic}(\bar{F})) / W$, where $O(\operatorname{Pic}(\bar{F}))$ is the orthogonal group of the Picard lattice of $\bar{F}$ and $W$ its normal subgroup generated by the reflections into the classes of nonsingular rational curves. For a "generic" Enriques surface $F$ this theorem allows to compute $\operatorname{Aut}(F)$ (see [3] and also [17], where this result is not stated explicitly). For an arbitrary $F$ the relation between $F$ and $\bar{F}$ does not help, since it is very difficult to compute the action of $\tau$ in $\operatorname{Pic}(\bar{F})$. However, by other means, we can prove the following analog of Piatetski-Shapiro and Shafarevich's result:

Theorem. Let $F$ be an Enriques surface and $H_{F}=\operatorname{Pic}(F) /$ Tors. Let $W_{F}^{n}$ be the subgroup of the orthogonal group $O\left(H_{F}\right)$ generated by reflections into the classes of nonsingular rational curves on $F$ and $G$ be the subgroup generated by $W_{F}^{n}$ and the image Aut $(F)^{*}$ of $\operatorname{Aut}(F)$ in $O\left(H_{F}\right)$. Then $W_{F}^{n}$ is a normal subgroup of $G$ and $G$ is the semi-direct product of $W_{F}^{n}$ and $\operatorname{Aut}(F)^{*}$. Moreover, if $k=\mathbb{C}$ then $G$ is of finite index in $O\left(H_{F}\right)$.

Our proof of this theorem depends upon the Global Torelli Theorem for Enriques surfaces proven by Horikawa in [12]. However, we use it differently than the similar theorem has been used in the case of K3-surfaces. As in the 
latter case we believe that the above theorem is true for $k \neq \mathbb{C}$. For example, we know that $\operatorname{Aut}(F)$ is always infinite if $W_{F}^{n}=\{1\}$ and finite if $W_{F}^{n}$ is of finite index in $O\left(H_{F}\right)$. An example of the latter situation is given in the paper. The Enriques surface from this example is not new, it was used by Horikawa in [12] for other aims. It also gives an example of an Enriques surface for which Aut $(F)$ does not act faithfully on the cohomology. Independently (and earlier) a similar example was constructed by W. Barth (see [3]).

This paper originates from an inspiring lecture of C. Peters about automorphisms of a "generic" Enriques surface. I owe to him for this very much. I also want to thank the Mathematics Institute of the University of Warwick and the Max Planck Institut für Mathematik in Bonn for providing the most stimulating atmosphere during the time when this paper was being written. I am very grateful to the referee for correcting a mistake in the proof of Theorem 4.3.

\section{§1. The double plane construction}

In this section we recall the classical construction of the presentation of an Enriques surface as a double cover of the projective plane $\mathbb{P}^{2}$ branched along the union of two lines and a special curve of degree 6 ([1], Chap. X). A better version of this construction is the presentation of an Enriques surface as a double cover of a 4-nodal Del Pezzo quartic surface or its degeneration branched along a nonsingular curve of degree 8 and the singular locus. This construction is well-known to the experts (see, for example, $[3,9,23]$ ). We state it without proof.

1.1. Proposition. Let $C$ be an irreducible curve on an Enriques surface $F$ with $C^{2}$ $=2$. Then the linear system $|2 C|$ is base-point-free and defines a morphism $\phi: F \rightarrow \mathbb{P}^{4}$ generically of degree 2 onto its image. The surface $S=\phi(\bar{F})$ is the intersection of two quadrics of rank 3 in $\mathbb{P}^{4}$ and is not a cone. The induced map $\phi: F \rightarrow S$ factors through a birational morphism $F \rightarrow F^{\prime}$ onto a surface with double rational points as singularities and a double cover $F^{\prime} \rightarrow S$ branched along the singular locus of $S$ and a curve $W$ cut by a quadric in $\mathbb{P}^{4}$.

1.2. Proposition. Let $S$ be an irreducible quartic surface in $\mathbb{P}^{4}$ which is given by two quadric equations of rank 3 and is not a cone over a curve in $\mathbb{P}^{3}$. Let $W \in\left|\mathcal{O}_{S}(2)\right|$ with at most $a_{n}, d_{n}, e_{n}$-singularities and not passing through the singular locus $\operatorname{Sing}(S)$ of $S$. Then there exists a unique double cover $F^{\prime} \rightarrow S$ branched along $W$ and $\operatorname{Sing}(S)$ such that $F^{\prime}$ has at most double rational points as singularities and a minimal resolution of $F^{\prime}$ is an Enriques surface $F$. The induced map $F \rightarrow S$ is given by a linear system $|2 C|$, where $C$ is an irreducible curve with $C^{2}=2$.

1.3. The following facts about the surfaces $S$ from above are well-known and can be easily proven. Up to a projective isomorphism there are only two such surfaces. The first is given by the equations

$$
x_{0}^{2}+x_{1}^{2}+x_{2}^{2}=0, \quad x_{0}^{2}+x_{3}^{2}+x_{4}^{2}=0 .
$$

It contains 4 ordinary double points $P_{ \pm}=(0,1, \pm i, 0,0)$ and $P_{ \pm}^{\prime}=(0,0,0,1, \pm i)$ as its singularities. It also contains the four lines $l_{ \pm}=\left\{x_{1} \pm i x_{2}=x_{3} \mp i x_{4}=x_{0}\right.$ 
$=0\}$ and $l_{ \pm}^{\prime}=\left\{x_{1} \mp i x_{2}=x_{3} \pm i x_{4}=x_{0}=0\right\}$ which form a quadrangle with the vertices at the points $P_{ \pm}, P_{ \pm}^{\prime}$. We will refer to this surface as a 4-nodal Del Pezzo quartic surface.

The second surface can be given by the equations:

$$
x_{0}^{2}+x_{1}^{2}+x_{2}^{2}=0, \quad x_{0} x_{4}+x_{3}^{2}=0 .
$$

It contains two ordinary double points $P_{ \pm}=(0,1, \pm i, 0,0)$ and a double rational point $P^{\prime}=(0,0,0,0,1)$ of type $A_{3}$ as its singularities. It also contains two lines $l_{ \pm}=\left\{x_{0}=x_{3}=x_{1} \pm i x_{2}=0\right\}$ which join the point $P^{\prime}$ with the point $P_{ \pm}$respectively. We will refer to this surface as a degenerate 4-nodal Del Pezzo quartic surface.

1.4. Let $\phi: F \rightarrow S$ be the morphism from Proposition 1.1. Suppose that $S$ is a 4nodal Del Pezzo quartic surface. Let $f_{ \pm}=\phi^{-1}\left(l_{ \pm}\right)$and $f_{ \pm}^{\prime}=\phi^{-1}\left(l_{ \pm}^{\prime}\right)$. One can easily see that $|2 C|=\left|f_{+}+f_{-}+f_{+}^{\prime}+f_{-}^{\prime}\right|, f_{+} \in\left|f_{-}+K_{F}\right|, f_{+}^{\prime} \in\left|f_{-}^{\prime}+K_{F}\right|,|f|=\left|2 f_{+}\right|$ $=\left|2 f_{-}\right|$and $\left|f^{\prime}\right|=\left|2 f_{+}^{\prime}\right|=\left|2 f_{-}^{\prime}\right|$ are irreducible pencils of elliptic curves with $f \cdot f^{\prime}$ $=4$. Conversely, if $|f|$ and $\left|f^{\prime}\right|$ are two irreducible pencils of elliptic curves on $F$ with $f \cdot f^{\prime}=4$, then $|f|$ (resp. $\left.\left|f^{\prime}\right|\right)$ contains exactly two multiple fibres $f_{ \pm}$ (resp. $f_{ \pm}^{\prime}$ ) such that $|f|=\left|2 f_{+}\right|=\left|2 f_{-}\right|$(resp. $\left|f^{\prime}\right|=\left|2 f_{+}^{\prime}\right|=\left|2 f_{-}^{\prime}\right|$ ) and the linear system $\left|2 f^{\prime}+2 f^{\prime}\right|=\left|f_{+}+f_{-}+f_{+}^{\prime}+f_{-}^{\prime}\right|$ is equal to $|2 C|$ for some irreducible curve $C$ with $C^{2}=2$. Thus, we see that the morphisms $\phi: F \rightarrow S$ to a fixed 4-nodal Del Pezzo quartic surface $S$ in $\mathbb{P}^{4}$ with the property of Propositions 1.1 and 1.2 are in $(1-1)$-correspondence with the pairs $\left(f, f^{\prime}\right)$ as above. We will call such a pair a standard pair on $F$.

Now we assume that $S$ is a degenerate 4-nodal Del Pezzo quartic. In this case $\phi^{-1}\left(l_{+} \cup l_{-}\right)=f_{+}+f_{-}+E$, where $|f|=\left|2 f_{+}\right|=\left|2 f_{-}\right|$is an irreducible elliptic pencil and $E$ a nonsingular rational curve such that $f \cdot E=2$. Conversely, if $(f, E)$ is a pair of curves as above, then the linear system $|2 f+2 E|$ is equal to $|2 C|$ for some irreducible curve $C$ with $C^{2}=2$. Thus, we see that the morphisms $\phi: F \rightarrow S$ to a fixed degenerate 4-nodal Del Pezzo quartic surface with the properties of Propositions 1.1 and 1.2 are in a $(1-1)$-correspondence with the $(|f|, E)$ as above. We will call such a pair a degenerate standard pair on $F$.

1.5. Let $S$ be a (degenerate) 4-nodal Del Pezzo quartic surface and $U \subset\left|\mathcal{O}_{S}(2)\right|$ be the open subvariety parametrizing the curves with at most $a_{n}, d_{n}, e_{n}$-singularities (i.e. neither infinitely near triple points nor points of multiplicity $\geqq 4$ occur) which do not pass through the singular locus of $S$. The group Aut $(S)$ acts regularly on $U$. One can check that $U$ is contained in the set of semistable points for the action of Aut $(S)$ in $\left|\mathcal{O}_{S}(2)\right|$ (see [13], where it is done for an equivalent problem). We denote the quotient variety by $\mathbf{M}_{E}^{n s}$ if $S$ is a 4nodal Del Pezzo quartic and by $\mathbf{M}_{E}^{s}$ if $S$ is a degenerate 4-nodal Del Pezzo quartic. One immediately computes $\operatorname{dim} \operatorname{Aut}(S)$ to deduce that $\operatorname{dim} \mathbf{M}_{E}^{n s}=10$ and $\operatorname{dim} \mathbf{M}_{E}^{s}=9$. By 1.4 we see that the points of $\mathbf{M}_{E}^{n s}\left(\right.$ resp. $\left.\mathbf{M}_{E}^{s}\right)$ are in a $(1-1)$ correspondence with the isomorphism classes of standard (resp. degenerate standard) pairs on Enriques surfaces. Here a pair $\left(|f|,\left|f^{\prime}\right|\right)($ resp. $(|f|, E))$ on a surface $F$ is called isomorphic to a pair $\left(|\bar{f}|, \bar{f}^{\prime} \mid\right)\left(\right.$ resp. $(|\vec{f}|, \bar{E})$ on a surface $F^{\prime}$ if there exists an isomorphism $\gamma: F^{\prime} \rightarrow F$ such that $\gamma^{*}\left\{|f|,\left|f^{\prime}\right|\right\}=\left\{|\bar{f}|,\left|\bar{f}^{\prime}\right|\right\}$ (resp. $\gamma^{*}\{|f|, E\}=\{|\bar{f}|, \bar{E}\}$ ). 
1.6. Let $\left(|f|,\left|f^{\prime}\right|\right)$ (resp. $\left.(|f|, E)\right)$ be a standard (resp. degenerate) pair on $F$. Let $H_{F}=\operatorname{Pic}(F) /$ Tors. For every element $D \in \operatorname{Pic}(F)$ we denote by $[D]$ the class of $D$ in $H_{F}$. Let $H_{F}^{+}$be the set of the classes of effective divisors on $F$ in $H_{F}$. There exists a unique pair $\left(x_{1}, x_{2}\right) \in H_{F}$ such that $\left([f],\left[f^{\prime}\right]\right)=\left(2 x_{1}, 2 x_{2}\right)$ (resp. $\left.([f],[E])=\left(2 x_{1}, x_{1}-x_{2}\right)\right)$ and $x_{1}^{2}=x_{2}^{2}=0, x_{1} \cdot x_{2}=1, x_{i} \cdot y \geqq 0$ for $i=1,2$ and all $y \in H_{F}^{+}$(resp. $x_{1} \cdot y \geqq$ for all $y \in H_{F}^{+}, x_{2}=x_{1}+[E]$ for some irreducible curve $E$ ). Indeed, it is well-known that every irreducible elliptic pencil on an Enriques surface has exactly two multiple fibres of multiplicity 2 (see, for example, [4]). Thus, we may take $x_{1}=\frac{1}{2}[f], x_{2}=\frac{1}{2}\left[f^{\prime}\right]$ (resp. $x_{2}=x_{1}+[E]$ ). The converse follows from the following

1.7. Lemma. Let $D$ be a divisor on $F$ with $D^{2} \geqq 0$. Then $|D|$ or $|-D|$ is not empty. Moreover, if $D^{2}=0, D \cdot C \geqq 0$ for any curve $C$, and $D \cdot D^{\prime}=1$ for some divisor $D^{\prime}$, then $|2 D|$ is an irreducible pencil of elliptic curves.

Proof. Standard, therefore omitted (see, for example, [4] and use [14], where it is proven that $F$ is not quasi-elliptic).

1.8. A pair $\left(x_{1}, x_{2}\right)$ from 1.6 defines an embedding of the unimodular even hyperbolic lattice $U=\mathbb{Z} v_{1} \oplus \mathbb{Z} v_{2}, v_{1}^{2}=v_{2}^{2}=0, v_{1} \cdot v_{2}=1$, into the lattice $H_{F}$ by sending $v_{i}$ to $x_{i}$. Conversely, if $F$ does not contain nonsingular rational curves, then every such embedding defines a pair $\left(x_{1}, x_{2}\right)$ from 1.6. This follows immediately from Lemma 1.7. If $F$ contains nonsingular rational curves, then the situation is more delicate. Let $W_{F}^{n}$ be the subgroup of the orthogonal group $O\left(H_{F}\right)$ which is generated by the reflections

$$
s_{E}: x \rightarrow x+(x \cdot[E])[E]
$$

where $E$ is a nonsingular rational curve. The group $O\left(H_{F}\right)$ and hence $W_{F}^{n}$ acts naturally on the set of the lattice embeddings $j: U \rightarrow H_{F}$. Also $O(U)$ acts on this set.

\subsection{Proposition. The correspondence}

a standard (degenerate) pair on $F \rightarrow$ an embedding $j: U \rightarrow H_{F}$

described in 1.7 defines a (1-1)-correspondence between the set of standard (degenerate) pairs on $F$ and the $O(U) \times W_{F}^{n}$-orbits in the set of embeddings $j: U \rightarrow H_{F}$.

Proof. Let $j: U \rightarrow H_{F}$ be an embedding and $\left(x_{1}, x_{2}\right)=\left(j\left(v_{1}\right), j\left(v_{2}\right)\right)$. Applying $O(U)$ we may assume that $x_{i} \in H_{F}^{+}$. We will show that after applying an element of $W_{F}^{n}$ we get a pair $\left(x_{1}^{\prime}, x_{2}^{\prime}\right)$, where $x_{1}^{\prime} \cdot y \geqq 0$ for all $y \in H_{F}^{+}$. Applying Lemma 1.7 we find an irreducible elliptic pencil $|f|$ such that $[f]=2 x_{1}$. Let $x_{2}=[D]$ for some curve $D$ and $D=\sum n_{i} C_{i}$ be its decomposition into irreducible components. Since $x_{1} \cdot[D]=\sum n_{i}\left(x_{1} \cdot\left[C_{i}\right]\right)=1$, we obtain that there exists a unique component $C_{i}$, say $C_{1}$, such that $x_{1} \cdot\left[C_{1}\right]=1, n_{1}=1$ and $x_{1} \cdot\left[C_{i}\right]=0$ for all $i \neq 1$. It will follow from the lemma below that after applying an element $w \in W_{F}^{n}$ to $[D]$ we may assume that $D \cdot C_{i} \geqq 0$ for any nonsingular rational curve $C_{i}, i \neq 1$, without changing $x_{1}$. Then, since $D^{2}=0$, we obtain that $C_{i}^{2}=0$ or -2 for any 
i. If $D \cdot C_{1} \geqq 0$, then we can apply Lemma 1.7 and put $x_{2}=[D]$. If $D \cdot C_{1}<0$, then

$$
0 \leqq x_{1} \cdot s_{C_{1}}([D])=[D] \cdot x_{1}+\left(D \cdot C_{1}\right)\left(x_{1} \cdot\left[C_{1}\right]\right)=1+D \cdot C_{1}
$$

(we will show in the next lemma that $s_{C_{1}}([D]) \in H_{F}^{+}$). This shows that $D \cdot C_{1}=$ -1 . Thus $s_{C_{1}}([D])=[D]-\left[C_{1}\right] \in H_{F}^{+}$and $x_{1} \cdot s_{C_{1}}([D])=0$. This easily implies that $[D]=\left[C_{1}\right]+x_{1}$ and we can put $x_{2}=[D]$. Thus, we have found that the $O(U) \times W_{F}^{n}$-orbit of $j$ contains an embedding coming from a standard (degenerate) pair. It remains to prove the promised lemma which will also imply the injectivity of the stated correspondence.

1.10. Lemma. The following properties of the action of $W_{F}^{n}$ in $H_{F}$ are true:

i) $W_{F}^{n}$ leaves the set $V_{F}^{+}=\left\{x \in H_{F}^{+}: x^{2} \geqq 0\right\}$ invariant;

(ii) the set $C_{F}^{n}=\left\{x \in V_{F}^{+}: x \cdot[E] \geqq 0\right.$ for every nonsingular rational curve $\left.E\right\}$ intersects every orbit of $W_{F}^{n}$ in $V_{F}^{+}$at exactly one element;

(iii) if $x \in V_{F}^{+}$is a fixed point of some $w \in W_{F}^{n}, w \neq 1$, then $x \cdot[E]=0$ for some nonsingular rational curve $E$.

Proof. (i) Let $x \in V_{F}^{+}$and $s_{E}(x) \notin V_{F}^{+}$for some nonsingular rational curve $E$. By Lemma $1.7-s_{E}(x)=-(x \cdot[E])[E]-x \in V_{F}^{+}$. This is obviously absurd because $|m E|=\emptyset$ or isolated for any integer $m$.

(ii) Let $x \in V_{F}^{+}$and $I_{x}=\{$ nonsingular rational curves $E$ such that $x \cdot[E]<0\}$. If $I_{x}=\emptyset$, then $x \in C_{F}^{n}$. Assume that $I_{x} \neq \emptyset$ and let $E \in I_{x}$. Then

$$
s_{E}(x) \cdot x_{0}=x \cdot x_{0}+\left(x \cdot x_{0}\right)\left(x_{0} \cdot[E]\right)<x \cdot x_{0},
$$

where $x_{0}$ is the class of an ample divisor on $F$. If $I_{s_{E}(x)} \neq \emptyset$ we do it again. Since $x_{0}$ intersects positively every element of $H_{F}^{+}$, this process must stop. Thus for a certain sequence $E_{1}, \ldots, E_{k}$ we get $I_{w(x)}=\emptyset$, where $w=s_{E_{k}} \circ \ldots \circ s_{E_{1}}$. This shows that $w(x) \in C_{F}^{n}$. It remains to prove the uniqueness property. Let $x^{\prime}$ $=w(x) \in C_{F}^{n}, x \in C_{F}^{n}, w=s_{E_{k}} \circ \ldots \circ s_{E_{1}}$ be a reduced decomposition of $w$ (see [6], Chap. IV, $\left.\S 1, n^{\circ} 1\right)$. We will prove that $x^{\prime}=x$ by induction on $l(w)=k$. If $l(w)$ $=1$, then $x^{\prime} \cdot\left[E_{1}\right]=-x \cdot\left[E_{1}\right]$ shows that $x \cdot\left[E_{1}\right]=0$ and hence $x^{\prime}=s_{E_{1}}(x)=x$. Let $w=s_{E_{k}} \circ w^{\prime}, \quad l\left(w^{\prime}\right)=k-1$. Then by [15], Proposition $1.5, w^{\prime}\left(C_{F}^{n}\right)$ $\subset\left\{x \in V_{F}^{+}: x \cdot\left[E_{k}\right] \geqq 0\right\}$ (though our situation is a little different, our root base is infinite, the proof goes through in the same way). Thus

$$
w(x) \cdot\left[E_{k}\right]=\left(w^{\prime}(x)+\left(w^{\prime}(x) \cdot\left[E_{k}\right]\right)\left[E_{k}\right]\right) \cdot\left[E_{k}\right]=-w^{\prime}(x) \cdot\left[E_{k}\right] \leqq 0
$$

and hence $w^{\prime}(x) \cdot\left[E_{k}\right]=0$ and $w(x)=w^{\prime}(x)$. By induction we get $w^{\prime}(x)=x$.

(iii) Follows from the proof of (ii).

1.11. Definition. A $U$-marked Enriques surface is a pair $(F, j)$, where $F$ is an Enriques surface and $j: U \rightarrow H_{F}$ an embedding of the lattices. An isomorphism between $U$-marked surfaces $(F, j)$ and $\left(F^{\prime}, j^{\prime}\right)$ is a triple $(f, \sigma, w)$, where $f: F \rightarrow F^{\prime}$ is an isomorphism of surfaces, $\sigma \in O(U), w \in W_{F}^{n}$ such that $j \circ \sigma=w \circ f^{*} \circ j^{\prime}$.

It follows from Proposition 2.1 that every Enriques surface $F$ admits a $U$ marking. 
1.12. Corollary. Let $\mathbf{M}_{E}=\mathbf{M}_{E}^{n s} \Perp \mathbf{M}_{E}^{s}$. Then the correspondence between the points of $\mathbf{M}_{E}$ and standard (degenerate) pairs on Enriques surfaces defines via 1.6 a bijection between the set $\mathbf{M}_{E}$ and the set of isomorphism classes of $U$-marked Enriques surfaces.

1.13. In the next section we will prove by using transcendental methods that the forgetful map

$\{U$-marked Enriques surfaces/isomorphism $\} \rightarrow\{$ Enriques surfaces/isomorphism $\}$

has finite fibres if $k=\mathbb{C}$. The extension of this property to the case $\operatorname{char}(k) \neq 0$ is the only obstacle for the validity of the main theorem (see Introduction) in the case $\operatorname{char}(k) \neq 0$.

\section{§2. The periods of Enriques surfaces}

2.1. Proposition. Let $F$ be an Enriques surface. Then $H_{F}=\operatorname{Pic}(F) /$ Tors is an even unimodular lattice of rank 10 and signature $(1,9)$. In particular, $H_{F}$ is isomorphic to the lattice $U \perp E_{8}$, where

$$
\begin{aligned}
& U=\mathbb{Z} v_{1} \oplus \mathbb{Z} v_{2}, \quad v_{1}^{2}=v_{2}^{2}=0, \quad v_{1} \cdot v_{2}=1, \\
& E_{8}=\mathbb{Z} e_{1} \oplus \ldots \oplus \mathbb{Z} e_{8}, \quad e_{i}^{2}=-2, i=1, \ldots, 8, e_{3} \cdot e_{8}=1, \\
& e_{1} \cdot e_{2}=e_{2} \cdot e_{3}=\ldots=e_{6} \cdot e_{7}=1, \quad e_{i} \cdot e_{j}=0 \text { otherwise. }
\end{aligned}
$$

Proof. If $k=\mathbb{C}$, this is well-known. By the Hodge decomposition, we have $\operatorname{Pic}(F)=H^{2}(F, \mathbb{Z}), H^{1}(F, \mathbb{Z})=$ Tors. By the formula $12\left(1-q+p_{g}\right)=K_{F}^{2}+c_{2}$, we get $H^{2}(F, \mathbb{Z}) /$ Tors $=\mathbb{Z}^{10}$. By Lefschetz, Tors $(\operatorname{Pic}(F))=\operatorname{Tors}\left(H^{2}(F, \mathbb{Z})\right.$. By Poincare, $H_{F}=H^{2}(F, \mathbb{Z})$ /Tors is a unimodular lattice. It is even, because $\operatorname{Pic}(F) /$ Tors is even. By Hodge Index Theorem, $H_{F}$ has signature $(1,9)$. The last assertion follows from the well-known classification of even indefinite unimodular lattices. If $\operatorname{char}(k)=p \neq 0$, this is less trivial. Using the fact that $F$ has an elliptic fibration (by [14] $F$ is not quasi-elliptic), one can prove that $\operatorname{rk}\left(H_{F}\right)=10([4]$, Th. 4). Again, by Hodge Index Theorem, $\operatorname{sign}\left(H_{F}\right)=(1,9)$. Since $2 K_{F}=0, H_{F}$ is an even lattice. So, it remains to prove that $H_{F}$ is unimodular. Comparing the Brauer group $\operatorname{Br}(F)=H^{2}\left(F, \mathbb{G}_{m}\right)$ of $F$ with the Brauer group of its Jacobian elliptic surface (which is rational), we easily see that $\operatorname{Br}(F)=\mathbb{Z} / 2$ (cf. [2], p. 557). Using the Kummer exact sequence for flat cohomology, we obtain that

$$
H_{F} \otimes \mathbb{Z}_{s} \simeq \lim _{\longleftarrow} H^{2}\left(F_{\mathrm{fl}}, \mu_{\mathrm{s}^{n}}\right) / \text { Tors }
$$

for any prime $s$. By the duality for flat cohomology of a surface [16], we get that $H_{F} \otimes \mathbb{Z}_{s}$ is nondegenerate for every $s$. This proves that $H_{F}$ is unimodular. Another proof can be given by using the crystalline cohomology instead of flat cohomology (see [21], where the similar argument is used for proving that the Picard lattice of a supersingular K 3-surface is p-elementary). Along the same lines the proposition is proven in L. Illusie, Complexe de de Rham-Witt et cohomologie cristalline. Ann. Ec. Norm Sup., 12, 501-661 (1979). 

C.

From now on in this section the base field is the field of complex numbers

2.2. Let $X$ be a $\mathrm{K} 3$-surface. It is known (see $[1]$, Chap. IX) that $H^{2}(X, \mathbb{Z})$ is isomorphic as a lattice to the lattice $U \perp U \perp U \perp E_{8} \perp E_{8}$, where $U$ and $E_{8}$ are the same as in 2.1. We denote the latter lattice by $L_{\mathrm{K} 3}$. We fix a basis of $L_{\mathrm{K} 3}$ of the form $v_{1}, v_{2}, v_{1}^{\prime}, v_{2}^{\prime}, v_{1}^{\prime \prime}, v_{2}^{\prime \prime}, e_{1}^{\prime}, \ldots, e_{8}^{\prime}, e_{1}^{\prime \prime}, \ldots, e_{8}^{\prime \prime}$, where the first three pairs are the standard bases of $U$ and the remaining two octuples are the standard bases of $E_{8}$ (as in 2.1).

Let $\theta: L_{\mathrm{K} 3} \rightarrow L_{\mathrm{K} 3}$ be the involution given by the formula

$$
\begin{gathered}
\theta\left(v_{i}\right)=v_{i}, \theta\left(v_{i}^{\prime}\right)=v_{i}^{\prime \prime}, \theta\left(v_{i}^{\prime \prime}\right)=v_{i}^{\prime}, \quad i=1,2, \\
\theta\left(e_{i}^{\prime}\right)=e_{i}^{\prime \prime}, \theta\left(e_{i}^{\prime \prime}\right)=e_{i}^{\prime}, \quad i=1, \ldots, 8 .
\end{gathered}
$$

Then the $\theta$-invariant sublattice is

$$
L_{\mathbf{K} 3}^{+}=\mathbb{Z} y_{1} \oplus \mathbb{Z} y_{2} \oplus \mathbb{Z} e_{1} \oplus \ldots \oplus \mathbb{Z} e_{8},
$$

where $y_{i}=v_{i}^{\prime}+v_{i}^{\prime \prime}, i=1,2$ and $e_{i}=e_{i}^{\prime}+e_{i}^{\prime \prime}, i=1, \ldots, 8$.

The $\theta$-anti-invariant sublattice is

$$
L_{\mathrm{K} 3}^{-}=\mathbb{Z} \bar{y}_{1} \oplus \mathbb{Z} \bar{y}_{2} \oplus \mathbb{Z} \bar{e}_{1} \oplus \ldots \oplus \mathbb{Z} \bar{e}_{8} \oplus \mathbb{Z} v_{1} \oplus \mathbb{Z} v_{2},
$$

where $\bar{y}_{i}=v_{i}^{\prime}-v_{i}^{\prime \prime}, i=1,2, \bar{e}_{i}=e_{i}^{\prime}-e_{i}^{\prime \prime}, i=1, \ldots, 8$.

Obviously,

$$
L_{\mathrm{K} 3}^{+} \simeq U(2) \oplus E_{8}(2), \quad L_{\mathrm{K} 3}^{-} \simeq U(2) \oplus E_{8}(2) \oplus U,
$$

where for every lattice $L$ and an integer $m L(m)$ denotes the lattice obtained from $L$ by multiplying its inner product by $m$.

Since $L_{\mathrm{K} 3} / L_{\mathrm{K} 3}^{ \pm}$does not have torsion, both of the lattices $L_{\mathrm{K} 3}^{+}$and $L_{\mathrm{K} 3}^{-}$are primitive sublattices of $L_{\mathrm{K} 3}$.

Let $F$ be an Enriques surface, $p: \bar{F} \rightarrow F$ its $\mathrm{K} 3$-cover and $\tau \in \operatorname{Aut}(\bar{F})$ the corresponding fixed point free involution of $\bar{F}$. It is possible to choose an isomorphism of lattices $\phi: H^{2}(\vec{F}, \mathbb{Z}) \rightarrow L_{\mathrm{K} 3}$ such that

$$
\phi \circ \tau^{*}=\theta \circ \phi
$$

(see [12], Thm. 5.1). Then, $\phi$ defines an isomorphism

$$
\bar{\phi}: H^{2}(\bar{F}, \mathbb{Z})^{\imath^{*}}=p^{*} H^{2}(F, \mathbb{Z}) \rightarrow L_{\mathrm{K} 3}^{+} .
$$

Using a non-vanishing holomorphic 2-form $\omega$ on $\bar{F}$, we define the period of $\bar{F}$, the line in $H^{2}(\bar{F}, \mathbb{C})$ spanned by $\omega$. Taking the image of this line under $\phi$, we get a point $P(\bar{F}, \phi)$ in the projective space $\mathbb{P}\left(L_{\mathrm{K} 3} \otimes \mathbb{C}\right)=\mathbb{P}^{21}$. Since $\omega$ is antiinvariant with respect to the involution $\tau$ (otherwise $H^{2}\left(F, \mathcal{O}_{F}\right) \neq 0$ ), the point $P(\bar{F}, \phi)$ belongs to the subspace $\mathbb{P}\left(L_{\mathrm{K} 3}^{-} \otimes \mathbb{C}\right)=\mathbb{P}^{11}$. Also, the Hodge bilinear relations $\omega \wedge \omega=0, \omega \wedge \bar{\omega}>0$ show that $P(\bar{F}, \phi)$ lies in an open subset $D$ of a nonsingular quadric in $\mathbb{P}^{11}$. The set $D$ is a disjoint union of two copies of a bounded symmetric domain of type IV associated to the group $S O(2,10)$. 
Let $O\left(L_{\mathrm{K} 3}\right)^{\theta}$ be the subgroup of the orthogonal group $O\left(L_{\mathrm{K} 3}\right)$ of the lattice $L_{\mathrm{K} 3}$ which consists of the isometries which commute with $\theta$. We have the canonical restriction homomorphisms

$$
r^{ \pm}: O\left(L_{\mathrm{K} 3}\right)^{\theta} \rightarrow O\left(L_{\mathrm{K} 3}^{ \pm}\right) .
$$

Let $\Gamma=r^{-}\left(O\left(L_{\mathrm{K} 3}\right)^{\theta}\right)$. The group $\Gamma$ is an arithmetic subgroup of $O(2,10)$, it acts discontinuously on $D$ and the quotient space $D / \Gamma$ is an irreducible algebraic variety ([12], p. 86). The image of the point $P(\bar{F}, \phi)$ in $D / \Gamma$ is independent of a choice of $\phi$. It is denoted by $P(F)$ and is called the period of $F$. According to [12] we have the Global Torelli Theorem for Enriques surfaces:

$$
F \simeq F^{\prime} \quad \text { if and only if } P(F)=P\left(F^{\prime}\right) .
$$

2.3. Theorem. The period map $(F, j) \rightarrow P(F)$ defines a quasi-finite morphism of algebraic varieties

$$
P: \mathbf{M}_{E}=\mathbf{M}_{E}^{n s} \Perp \mathbf{M}_{E}^{s} \rightarrow D / \Gamma
$$

Proof. We can prove it separately for $\mathbf{M}_{E}^{n s}$ and $\mathbf{M}_{E}^{s}$. We do the first part, the other is considered similarly. Let $U$ be the open subset of $\left|\mathcal{O}_{S}(2)\right|$ parametrizing the curves $B$ on a 4-nodal Del Pezzo quartic $S$ which do not pass through Sing $(S)$ and have singularities only of types $a_{n}, d_{n}, e_{n}$. Let $f^{\prime}: \mathscr{E}^{\prime} \rightarrow U$ be the family of double covers of $S$ branched along $B \in U$ and $\operatorname{Sing}(S)$ (i.e. the composition of the double cover of $U \times S$ branched along the universal curve plus $U \times \operatorname{Sing}(S)$ and the first projection map $U \times S \rightarrow S$ ). Let $f: \mathscr{E} \rightarrow \bar{U}$ be a simultaneous resolution of rational double points of the fibres of $f^{\prime}$. Recall that this means that there exist a finite Galois cover $q: \vec{U} \rightarrow U$ and a morphism $r: \underline{\bar{E}} \rightarrow \underline{\mathscr{E}}^{\prime}$ such that $f^{\prime} \circ r=q \circ f$ and the induced morphism $\mathscr{E}_{\tilde{u}} \rightarrow \mathscr{E}_{q(\bar{u})}^{\prime}$ is a minimal resolution of singularities. Let $\bar{p}: \underline{\vec{E}} \rightarrow \underline{\mathscr{E}}^{\prime}$ be the double cover corresponding to the sheaf $\omega_{\mathscr{E} / \mathrm{C}}$ and $\bar{f}: \overline{\mathscr{E}} \rightarrow \bar{U}$ be the composite map $f \circ p$. The fibres of $\bar{f}$ are nonsingular K3-surfaces, the double covers of the Enriques surfaces $\mathscr{E}_{\bar{u}}$. For any point $\bar{u} \in \bar{U}$ we can trivialize the sheaf $R^{2} \bar{f}_{*}\left(\mathbb{C}_{\tilde{g}}\right)$ in a neighborhood $V(\bar{u})$ of $\bar{u}$ and define the period map $V(\vec{u}) \rightarrow D$ as in 2.2. This shows that the period map $\bar{P}: \bar{U} \rightarrow D / \Gamma, \bar{u} \rightarrow P\left(\mathscr{E}_{\bar{u}}\right)$ is a holomorphic map locally liftable to $D$. By Borel's Extension Theorem ([5]) $\bar{P}$ is an algebraic map. Since $\bar{P}(\bar{u})$ is independent of a choice of a point $\bar{u}$ over $u, \bar{P}$ factors through $U$. Obviously, it factors also through $U \rightarrow U / \operatorname{Aut}(S)$. Thus, we have a morphism of algebraic varieties:

$$
P: U / \operatorname{Aut}(S)=\mathbf{M}_{E}^{n s} \rightarrow D / \Gamma .
$$

The fibres of $P$ are set-theoretically the isomorphism classes of $U$-marked Enriques surfaces $(F, j)$ with the fixed isomorphism class of $F$. Clearly, they are discrete. Therefore, the fibres of $P$ are finite.

2.4. Corollary. Let $F$ be an Enriques surface. Then the number of nonisomorphic embeddings $j: U \rightarrow H_{F}$ is finite.

2.5. Remark. The degree of the map $P$ was computed in [3]. It is equal to $2^{7} \cdot 17 \cdot 31$. It is known that $P\left(\mathbf{M}_{E}^{s}\right) \subset P\left(\mathbf{M}_{E}^{n s}\right)$ (F. Cossec). The variety $D / \Gamma$ is obviously unirational. It is not known whether it is rational $\left(\mathbf{M}_{E}^{n s}\right.$ is rational). 


\section{§3. The main theorem}

\subsection{Let}

$$
\rho: \operatorname{Aut}(F) \rightarrow O\left(H_{F}\right), \quad g \rightarrow g^{*}
$$

be the natural representation of the automorphism group $\operatorname{Aut}(F)$ of an Enriques surface in the lattice $H_{F}$. We denote by $\operatorname{Aut}(F)^{*}$ its image. The kernel $\operatorname{Ker}(\rho)$ is always finite because it leaves any very ample divisor invariant and hence must be isomorphic to a group of projective transformations of $F$. The latter is finite, since $H^{\mathrm{o}}\left(F, \theta_{F}\right)=H^{\mathrm{o}}\left(\bar{F}, \theta_{\bar{F}}\right)^{\mathrm{r}}=0$ (see [20]). If $k=\mathbb{C}$ then $\operatorname{Ker}(\rho)$ is trivial for a generic Enriques surface (see $[17], \S 10)$. We will show in $\S 4$ that $\operatorname{Ker}(\rho)$ may be non-trivial.

Let $G$ be the subgroup of $O\left(H_{F}\right)$ generated by the subgroups $\operatorname{Aut}(F)^{*}$ and $W_{F}^{n}$.

3.2. Proposition. $W_{F}^{n}$ is a normal subgroup of $G$ and $W_{F}^{n} \cap \operatorname{Aut}(F)^{*}=\{1\}$. In other words, $G$ is a semi-direct product of the subgroups $W_{F}^{n}$ and $\operatorname{Aut}(F)^{*}$.

Proof. For every nonsingular rational curve $E$ on $F$ and $g \in \operatorname{Aut}(F)$ we have

$$
g^{*} \circ s_{E} \circ g^{*-1}=s_{g(E)} .
$$

This shows that $W_{F}^{n}$ is normal in $G$. Let $x_{0} \in H_{F}$ be the class of an ample divisor. Clearly $x_{0} \in C_{F}^{n}$ and $g^{*}\left(x_{0}\right) \in C_{F}^{n}$ for every $g \in \operatorname{Aut}(F)$. It follows from Lemma 1.10 that $g^{*} \in W_{F}^{n}$ would imply that $x_{0} \cdot[E]=0$ for some nonsingular rational curve $E$. But this is impossible because $x_{0}$ comes from an ample divisor. Thus $\operatorname{Aut}(F)^{*} \cap W_{F}^{n}=\{1\}$ and the canonical homomorphism

is an isomorphism.

$$
\operatorname{Aut}(F)^{*} \rightarrow G / W_{F}^{n}
$$

3.3. Theorem. Assume that $k=\mathbb{C}$. Then $G$ is of finite index in $O\left(H_{F}\right)$.

Proof. Let $(F, j)$ be a $U$-marking of $F$. By the Witt theorem the group $O\left(H_{F}\right)$ acts transitively on the set of $U$-markings of $F$. Thus, the set of all $U$-marked Enriques surfaces $(F, j)$ ( $F$ is fixed) is bijective to the set of right cosets $O\left(H_{F}\right) / O\left(H_{F}\right)_{j}$, where $O\left(H_{F}\right)_{j}$ is the isotropy subgroup of $j$. The latter is isomorphic to the orthogonal group $O\left(j(U)^{\perp}\right)$ which is a finite because $j(U)^{\perp} \simeq E_{8}$. Now, the set of isomorphism classes of $U$-markings of $F$ is bijective to the set of double cosets $G \backslash O\left(H_{F}\right) / O(H)_{j}$ modulo an action of the finite group $O(U)$. Obviously, this set is finite if and only if $G$ is of finite index in $O\left(H_{F}\right)$. It remains to apply Corollary 2.4 .

3.4. Corollary. Let $F$ be an Enriques surface over $k=\mathbb{C}$. The following properties are equivalent:

(i) $\operatorname{Aut}(F)$ is finite;

(ii) $W_{F}^{n}$ is of finite index in $O\left(H_{F}\right)$.

Moreover, (ii) implies (i) for any $k$ of char $\neq 2$. 
3.5. Corollary. Let $F$ be an Enriques surface over $k=\mathbb{C}$. Suppose that $F$ does not contain nonsingular rational curves. Then $\operatorname{Aut}(F)$ is infinite.

Another proof of this fact valid for $k \neq \mathbb{C}$ is given in 4.1 .

3.6. Remark. It is known that $O\left(H_{F}\right)=W \cdot\{ \pm \mathrm{id}\}$, where $W$ is the reflection subgroup of $O\left(H_{F}\right)$, the subgroup generated by the reflections

$$
s_{e}: x \rightarrow x+(x \cdot e) e, \quad e^{2}=-2 .
$$

(see [10]). This shows that $\operatorname{Aut}(F)^{*}$ is always a subgroup of $W$. If $k=\mathbb{C}$ then for a generic $F$ the group $\operatorname{Aut}(F)$ is isomorphic to the 2-level congruence subgroup $W(2)$ of $W$, i.e. the subgroup of the elements of $W$ which act identically on $H_{F} / 2 H_{F}$ (see [3] and also [17], $\S 10$, where the result is not stated explicitly). The next result can be proven using the following purely algebraic fact which was stated in [7] without complete proof, and has been proven recently by $E$. Looijenga.

"Let $\sigma_{0}=\mathrm{id}_{U} \oplus-\mathrm{id}_{E_{8}} \in O\left(U \perp E_{8}\right)$. Then $W(2)$ is generated by the conjugates of $\sigma_{0}$ in $W^{\prime \prime}$.

3.7. Proposition. Suppose $F$ does not contain nonsingular rational curves. Then Aut $(F)^{*}$ contains $W(2)$.

Proof. Let $j: U \rightarrow H_{F}$ be an embedding. By 1.9 it defines a standard pair $\left(|f|,\left|f^{\prime}\right|\right)$ and the corresponding double cover $\phi(j): F \rightarrow S$, where $S$ is a 4-nodal Del Pezzo quartic surface. Let $\gamma(j) \in \operatorname{Aut}(F)$ be the corresponding involution of $F$. The fixed locus of $\gamma(j)$ is a nonsingular curve of genus 5 and four isolated points. By the Lefschetz fixed point formula $\operatorname{Tr}\left(\gamma(j)^{*} \mid H_{F}\right)=-6$. Obviously $\gamma(j)^{*}$ acts identically on $j(U)$. Hence $\operatorname{Tr}\left(\gamma(j)^{*} \mid j(U)^{\perp}\right)=-8$. On the other hand $\gamma(j)^{2}$ $=\mathrm{id}$ and $\operatorname{rk}\left(j(U)^{\perp}\right)=8$. This shows that $\gamma(j)^{*}$ acts as $-\mathrm{id}$ on $j(U)^{\perp}$ and as $\mathrm{id}_{j(U)}$ $\oplus\left(-\mathrm{id}_{j(U)}\right)$ on $H_{F}$. Choosing an isometry $\chi: H_{F} \rightarrow U \perp E_{8}$ such that $\chi(j(U))=U$, $\chi\left(j(U)^{\perp}\right)=E_{8}$, we may identify $\gamma(j)^{*}$ with the involution $\sigma_{0}$ from 3.6, i.e. $\chi^{-1}$ $\circ \gamma(j)^{*} \circ \chi=\sigma_{0}$. Replacing $j$ by an embedding $w \circ j, w \in W$, we get

$$
(w \circ j)^{*}=w \circ \gamma(j)^{* \circ w^{-1}}, \chi^{-1} \circ \gamma(w \circ j)^{*} \circ \chi=\left(\chi^{-1} \circ w \circ \chi\right) \circ \sigma_{0} \circ\left(\chi^{-1} \circ w \circ \chi\right)^{-1} \text {. }
$$

This shows that the isometry $\chi$ defines an isomorphism of $W(2)$ onto a subgroup of $\operatorname{Aut}(F)^{*}$.

3.8. Remark. The fact that a double cover $F \rightarrow S$ induces the involution $\sigma_{0}$ in $H_{F} \simeq U \perp E_{8}$ was pointed out to me by $C$. Peters. It has a striking analogy with the corresponding result for the double covers of Del Pezzo surfaces of degree 1 and 2 induced by the anti-bicanonical and anti-canonical linear system respectively. Namely, this involution induces - id on the sublattice of the Picard group consisting of the classes orthogonal to the canonical class. The latter is isomorphic to the lattice $E_{8}$ and $E_{7}$ respectively. It is known (see [6], Chap. VI, $\S 4$, Ex. 1 and 3) that $W\left(E_{8}\right)(2)$ and $W\left(E_{7}\right)(2)$ are equal to $\{ \pm \mathrm{id}$.

3.9. Remark. Let $Y$ be a rational surface obtained by blowing up 10 points in $\mathbb{P}^{2}$. It is easily seen that the orthogonal complement of the canonical class $K_{Y}$ in $\operatorname{Pic}(Y)$ is isomorphic to the lattice $U \perp E_{8}$. In the case when the 10 points 
are the ten double points of a rational curve of degree 6 one can develop the theory of the surfaces $Y$ which is quite similar to the theory of Enriques surfaces. In particular, using the ideas of [7], it can be shown that $\operatorname{Aut}(Y) \supset W(2)$ for generic $Y$. We hope to discuss the Coble surfaces $Y$ in a future paper.

3.10. Remark. If $k=\mathbb{C}$ we can prove by using the Global Torelli Theorem for K3-surfaces that $\operatorname{Aut}(F)^{*}$ is normal in $O\left(H_{F}\right)$ for generic $F$. Since the group $W / W(2) \simeq O^{+}\left(10, \mathbb{F}_{2}\right)($ see $[8,11])$ is almost simple (it contains a simple subgroup of index 2), Proposition 3.7 implies that $\operatorname{Aut}(F) \simeq W(2)$ for a generic $F$. The same statement is true for Coble surfaces.

\section{§4. An example}

In this section we give an example of an Enriques surface with finitely many automorphisms.

4.1. It is known that every Enriques surface $F$ has an irreducible pencil of elliptic curves which defines an elliptic fibration $f: F \rightarrow \mathbb{P}^{1}$ (see [4]). As we saw in $\S 1$ this fact follows from the existence of an embedding $j: U \rightarrow H_{F}$ and is used for proving that such embeddings exist in the case $\operatorname{char}(k) \neq 0$ (see Proposition 2.1). Let $f^{\prime}: J \rightarrow \mathbb{P}^{1}$ be the Jacobian fibration associated to $f$. It follows from the theory of elliptic surfaces (see [1], Chap. VI) that the group $J\left(\mathbb{P}^{1}\right)$ of sections of $f^{\prime}$ acts on $F$. In particular, $\operatorname{Aut}(F)$ is infinite if $J\left(\mathbb{P}^{1}\right)$ is infinite. The surface $J(F)$ is rational, it is obtained from $\mathbb{P}^{1}$ by blowing up the base points of a pencil of cubic curves. A well-known formula for the rank of $J\left(\mathbb{P}^{1}\right)$ (one can find it for example in [2]) implies that $J\left(\mathbb{P}^{1}\right)$ is finite only if the number of irreducible components in the union of the reducible fibres of $f^{\prime}$ (and hence of $f$ ) is equal to $8+k$, where $k$ is the number of the reducible fibres. This shows that $\operatorname{Aut}(F)$ is always infinite if $F$ does not contain nonsingular rational curves, because only they can be the irreducible components of the reducible fibres of $f$. This is another proof of Corollary 3.5, where $k$ is an arbitrary field of characteristic $\neq 2$. Also, it suggests that we have to look for an Enriques surface with finitely many automorphisms among the elliptic Enriques surfaces with "large" degenerate fibres.

4.2. Example. Let $F$ be an Enriques surface which has an elliptic fibration $f: F \rightarrow \mathbb{P}^{1}$ with two reducible fibres, one of type $\mathrm{III}^{*}\left(\right.$ or $\left.\tilde{E}_{7}\right)$ :

$$
f_{1}=E_{1}+2 E_{2}+3 E_{3}+4 E_{4}+2 E_{5}+3 E_{6}+2 E_{7}+E_{8}
$$

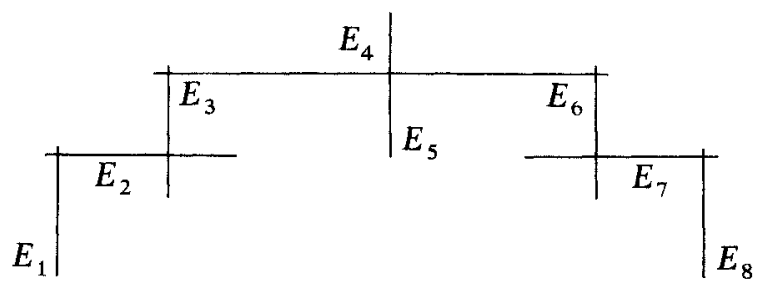


and one of type $I_{1}$ (or $\tilde{A}_{2}$ ) with multiplicity 2

$$
f_{2}=2 E_{1}^{\prime}+2 E_{2}^{\prime}
$$

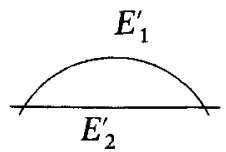

Also we need that $F$ contains a rational curve $E$ such that

$$
E \cdot E_{1}^{\prime}=E \cdot E_{1}=E \cdot E_{8}=1, E \cdot E_{2}^{\prime}=E \cdot E_{i}=0, \quad i \neq 1,8 .
$$

This is the only nontrivial property to realize, the existence of the two reducible fibres as above can be easily obtained by considering an appropriate pencil of cubic curves in $\mathbb{P}^{2}$. The existence of $F$ with the needed properties is shown in [12], p. 82.

4.3. Theorem. Let $F$ be an Enriques surface from Example 4.2. Then $\operatorname{Aut}(F)$ is finite.

Proof. Let $f_{0}=E+E_{1}+E_{2}+E_{3}+E_{4}+E_{6}+E_{7}+E_{8}$. Since $E_{5} \cdot f_{0}=1$, $\left[f_{0}\right]$ is a primitive isotropic vector. By Lemma $1.7\left|2 f_{0}\right|$ is an irreducible pencil of elliptic curves. Since $f_{0} \cdot E_{2}^{\prime}=0$, the rational curve $E_{2}^{\prime}$ is an irreducible component of a reducible fibre of $\left|2 f_{0}\right|$. This fibre cannot have more than two components because otherwise $r k\left(H_{F}\right)>10$. Let $E_{3}^{\prime}$ be the other component. It follows from the classification of elliptic fibres that $E_{3}^{\prime} \cdot E_{2}^{\prime}=2$. The curve $E_{5}$ does not intersect $E_{2}^{\prime}$, hence it intersects $E_{3}^{\prime}$. We have $E_{5} \cdot E_{3}^{\prime}=E_{1}^{\prime} \cdot E_{2}^{\prime}=2$. Summarizing, we have the following intersection graph $G(F)$ :

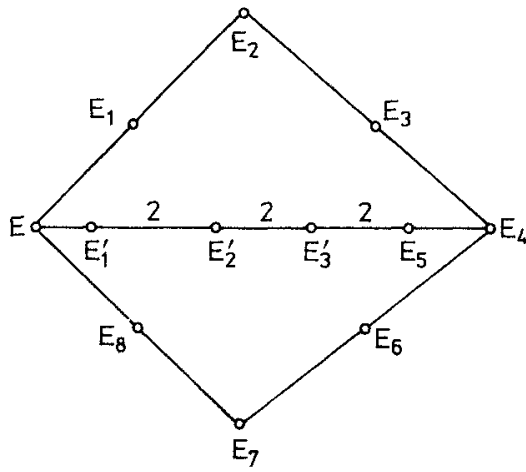

Let $W^{\prime}$ be the subgroup of $O\left(H_{F}\right)$ generated by the reflections into the classes of the curves from this graph. These generators define a structure of a Coxeter system on $W^{\prime}$. Its Coxeter graph is obtained from the above graph by replacing 2 by $\infty$. This Coxeter graph does not contain subgraphs corresponding to the Lanner groups and all its maximal parabolic subgraphs are of type $\tilde{E}_{8}, \tilde{D}_{8}, \tilde{A}_{7} \oplus \tilde{A}_{1}$ and $\tilde{E}_{7} \oplus \tilde{A}_{1}$ which all have the maximal possible rank. It follows from Theorem 2.6 bis of [24] that $W^{\prime}$ is of finite index in $O\left(H_{F}\right)$. Since $W^{\prime} \subset W_{F}^{n}$, we are done due to Corollary 3.4 . 
4.4. The automorphism group of the graph $G(F)$ from 4.3 is isomorphic to the group $\mathbb{Z} / 2 \oplus \mathbb{Z} / 2$. The elements of this group can be realized by automorphisms of $F$.

Let $g_{1}$ be the involution of $F$ defined by the standard degenerate pair $\left(\left|f_{1}\right|, E\right)$. The corresponding map $F \rightarrow S$ blows down the curves $E_{2}, \ldots, E_{7}$ to a $e_{6}$-point of the branch curve $B \subset\left|\mathcal{O}_{S}(2)\right|$ and blows down the curve $E_{2}^{\prime}$ to a node of $B$. It follows from the Jung resolution of the point $e_{6}$ that the involution $g_{1}$ switches the curves $E_{1}$ and $E_{8}, E_{2}$ and $E_{7}, E_{3}$ and $E_{6}$, and leaves the curves $E_{4}, \mathrm{E}_{5}, E_{1}^{\prime}, E_{2}^{\prime}$ invariant. The fixed point set of $g_{1}$ is the union of the curve $E_{5}$, a nonsingular elliptic curve $\bar{B}$ and four isolated points.

We see that $g_{1}$ induces an automorphism of $G(F)$ which is the reflection with respect to the middle line.

Let $f: F \rightarrow \mathbb{P}^{1}$ be the elliptic fibration induced by the pencil $\left|2 f_{0}\right|$. The Jacobian fibration $f^{\prime}: F^{\prime} \rightarrow \mathbb{P}^{1}$ has a section of order 2 which acts by translation on $F$ sending the curve $E_{5}$ to the curve $E_{1}^{\prime}$. Let $g_{2}$ be the corresponding involution of $F$. It is easy to see that $g_{2}$ induces an automorphism of $G(F)$ which is the rotation about $180^{\circ}$. Together with $g_{1}$ the involution $g_{2}$ generates the automorphism group of the diagram.

Let $g_{0}$ be the involution of $F$ defined by the degenerate standard pair $\left(\left|f_{1}^{\prime}\right|, E_{2}^{\prime}\right)$, where $f_{1}^{\prime}=E_{1}^{\prime}+2 E+3 E_{8}+4 E_{7}+5 E_{6}+6 E_{4}+3 E_{5}+4 E_{3}$. The corresponding map $F \rightarrow S$ blows down the curves $E_{2}, \ldots, E_{8}, E$ to a $e_{8}$-point of the branch curve $\bar{B}^{\prime} \subset\left|\mathcal{O}_{S}(2)\right|$. The fixed point set of $g_{0}$ consists of the curves $E, E_{2}, E_{4}, E_{7}$, a nonsingular elliptic curve $\bar{B}^{\prime}$ and four isolated points. One easily sees that $g_{0}$ leaves all the curves $E_{i}, E$ and $E_{i}^{\prime}$ invariant. Hence $g_{0}^{*}=\mathrm{id}_{H_{F}}$. The curve $\bar{B}^{\prime \prime}$ intersects $E_{5}$ transversally at one point. Therefore, $g_{0}$ has fixed point on all fibres of $\left|f_{1}^{\prime}\right|$ and cannot exchange the two reduced fibres of $f_{1}^{\prime}$. This immediately implies that

$$
\mathrm{g}_{0}^{*}=\mathrm{id}_{\mathrm{Pic}(F)} .
$$

In particular, $g_{0}$ induces the trivial automorphism of the graph $G(F)$.

It follows from [24], p. 334, that the vertices of the graph $G(F)$ represent the set of all nodal curves on $F$. Thus, the group Aut $(F)$ acts on $G(F)$. As we saw above, the corresponding homomorphism

$$
r: \operatorname{Aut}(F) \rightarrow \operatorname{Aut}(G(F))=\mathbb{Z} / 2 \oplus \mathbb{Z} / 2
$$

is surjective and $g_{0} \in \operatorname{Ker}(r)$.

Let $g_{0}^{\prime}$ be a nontrivial element of $\operatorname{Ker}(r)$. Since it leaves the curves $E, E_{1}, E_{8}$ and $E_{1}^{\prime}$ invariant, it must fix the points $E \cap E_{1}, E \cap E_{8}$ and $E \cap E_{1}^{\prime}$. This immediately implies that $g_{0}^{\prime}$ fixes the whole curve $E$ pointwise (a nontrivial automorphism of finite order of $\mathbb{P}^{1}$ has 2 fixed points). Since the set $E_{1}^{\prime} \cap E_{2}^{\prime}$ is $g_{0}^{\prime}$ invariant, $g_{0}^{\prime 2}$ has at least three fixed points on $E_{1}^{\prime}$. This implies that $g_{0}^{\prime 2}$ fixes $E_{1}^{\prime}$ pointwise. However, the fixed point set of any automorphism of $F$ of finite order is smooth. Since $E$ intersects $E_{1}^{\prime}$, we obtain that $g_{0}^{\prime 2}=\mathrm{id}_{F}$. In the same way, we see that $g_{0}^{\prime}$ cannot fix $E_{1}$ pointwise. Thus, $g_{0}\left|E_{1}=g_{0}^{\prime}\right| E_{1}$ and we get that $g_{0} \circ g_{0}^{\prime}$ fixes $E \cup E_{1}$ pointwise. This again implies that $g_{0}^{\prime} \circ g_{0}=\mathrm{id}_{F}$. Hence $g_{0}=g_{0}^{\prime}$ and

$$
\operatorname{Ker}(r)=\left(g_{0}\right) \simeq \mathbb{Z} / 2 \text {. }
$$


Let us show that $g_{1}$ and $g_{2}$ do not commute. For this we notice that $E_{5}$ is fixed pointwise by $g_{1}$ but $E_{1}^{\prime}=g_{2}\left(E_{5}\right)$ is not. This obviously implies that $g_{2} \circ g_{1} \circ g_{2} \neq g_{1}$. Thus, we get

and

$$
\left(g_{1} \circ g_{2}\right)^{2}=g_{0}
$$$$
\operatorname{Aut}(F) \simeq D_{4},
$$

the dihedral group of order 8 .

By other method the same result was obtained in [3].

4.5. Proposition. Let $\vec{F}$ be the $\mathrm{K} 3$-cover of the Enriques surface $F$ from Example 4.2. Assume that $k=\mathbb{C}$. Then Aut $(\bar{F})$ is infinite.

Proof. Let $p: \bar{F} \rightarrow S$ be the K3-cover. The pull-back of the elliptic pencil $\left|f_{1}\right|$ is an elliptic pencil on $\bar{F}$ with two degenerate fibres of type $E_{7}$ and a fibre of type $A_{3}$ (we use that $p$ splits over the fibre $f_{1}$ and does not split over the fibre $f_{2}$ ). The pull back of the 2-section $E$ splits under $p$ into two disjoint sections of this pencil. This easily implies that $\operatorname{rk}(\operatorname{Pic}(\bar{F}))=19$ or 20 . In the latter case $\operatorname{Aut}(\bar{F})$ must be infinite (see [22] or [17]). If $\operatorname{rk}(\operatorname{Pic}(\bar{F}))=19$ and $\operatorname{Aut}(\bar{F})$ is finite, then $\operatorname{Pic}(\bar{F}) \simeq A_{1} \perp E_{8} \perp E_{8} \perp U$ ([17], Thms. 0.2.3 and 0.2.4). Replacing the pencil $\left|f_{1}\right|$ by the pencil $\left|f_{1}^{\prime}\right|$ from 4.4 and arguing as above, we see that $\operatorname{Pic}(\bar{F})$ contains the lattice $L=E_{8} \perp E_{8} \perp U(2)$. Passing to the orthogonal complements of the lattices $\operatorname{Pic}(\bar{F})$ and $L$ in the lattice $L_{\mathrm{K} 3}=H^{2}(\bar{F}, \mathbb{Z})$, we see that $\left(A_{1}\right)_{U}^{\perp}$ is contained in $(U(2))_{U \perp U}^{\perp} \simeq U(2)$. This is absurd because $U(2)$ does not represent 2 .

4.6. Remark. The lattice $L$ from above can be characterized as the sublattice of $\operatorname{Pic}(\bar{F})$ spanned by the cycles which are invariant with respect to one of the two liftings to $\bar{F}$ of the involution $g_{0}$ which acts trivially on $\operatorname{Pic}(F)=H^{2}(F, \mathbb{Z})$. This was shown by $\mathrm{S}$. Mukai who also proved that the involution of the Enriques surface $F$ from Example 3 of [18] acts trivially on $\operatorname{Pic}(F)$ and its lifting to $\vec{F}$ fixes a sublattice $L$ isomorphic to the lattice $(U(2) \perp U(2))_{L_{\mathrm{K} 3}}^{\perp} \simeq D_{8} \perp D_{8} \perp U$. Recall that in our case $L \simeq(U \perp U(2))_{L_{\mathrm{K} 3}}^{\perp}$. Recently S. Mukai and Y. Namikawa have classified the periods of the Enriques surfaces $F$ whose automorphism group does not act faithfully on $H_{F}$. It follows from this result that the above two lattice $L$ are the only possible sublattices of $\operatorname{Pic}(\bar{F})$ spanned by the cycles which are invariant with respect to a lifting of an involution of $F$ acting trivially on $H_{F}$. Notice that the first example of an Enriques surface $F$ whose automorphism group does not act faithfully on Pic $(F)$ was constructed by W. Barth (see [3]). The impossibility of this was erroneously claimed in [25]. The error was pointed out later by C. Peters.

4.7. Conjecture. Let $F$ be an Enriques surface and $\bar{F}$ be its $\mathrm{K} 3$-cover. Then Aut $(\bar{F})$ is infinite.

\section{References}

1. Algebraic surfaces (ed. by I.R. Shafarevich). Proc. of Steklov Math. Inst., t. 75, Moscow, 1965 (Engl. Translation by A.M.S.)

2. Artin, M.: Supersingular K3 surfaces. Ann. Scient. École Norm. Sup. (4e) 7, 543-568 (1974) 
3. Barth, W., Peters, C.: Automorphisms of Enriques surfaces. Invent. math. 73, 383-411 (1983)

4. Bombieri, E., Mumford, D.: Enriques' classification of surfaces in Char. p. III. Invent. Math. 35, 197-232 (1976)

5. Borel, A.: Some metric properties of arithmetic quotients of bounded symmetric spaces and an extension theorem. J. Diff. Geom. 6, 543-560 (1972)

6. Bourbaki, N.: Groupes et algebrès de Lie. Paris: Hermann, 1968

7. Coble, A.: The ten nodes of the rational sextic and of the Cayley symmetroid. Amer. J. Math. 41, 243-265 (1919)

8. Coble, A.: Theta modular groups determined by point sets. Amer. J. Math. 40, 317-340 (1918)

9. Cossec, F.: Projective models of Enriques surfaces. Math. Ann. 265, 283-334 (1983)

10. Du Val, P.: On the Kantor group of a set of points in a plane. Proc. London Math. Soc. (2) 35 , 23-74 (1932)

11. Griess, R.: Quotients of infinite reflection groups. Math. Ann. 263, 267-278 (1983)

12. Horikawa, E.: On the periods of Enriques surfaces. I. Math. Ann. 234, 73-88 (1978)

13. Horikawa, E.: On the periods of Enriques surfaces. II. Math. Ann. 235, 217-246 (1978)

14. Lang, W.: On quasi-elliptic surfaces in Char. 3, Ann. Scient. École Norm. Sup. (4 $\left.{ }^{\mathrm{e}}\right)$ 12, 473-500 (1979)

15. Looijenga, E.: Invariant theory for generalized root systems. Invent. Math. 61, 1-32 (1980)

16. Milne, J.: Duality in the flat cohomology of a surface. Ann. Scient. École Norm. Sup. (4⿻) 9 , 171-201 (1976)

17. Nikulin, V.: On the quotient groups of the automorphism groups of hyperbolic forms by the subgroups generated by 2-reflections. Algebraic-geometrical applications. Modern Problems of Mathematics, t. 18, 1-114, Moscow, VINITI. 1981 (Engl. Transl.: J. Soviet. Math. 22, 1401$1476(1983))$

18. Peters, C.: On automorphisms of compact Kähler surfaces. Journ. Géom. Algébrique d'Angers (Beauville, A. ed.), Sijthoff \& Noordhoff 1980, pp. 249-267

19. Piatecky-Shapiro, I., Shafarevich, I.: A Torelli theorem for algebraic surfaces of type K3. Izvestia AN SSSR, Ser. Math. 35, 530-572 (1971); (Engl. Transl.: Math. USSR-Izvestia 5, 547-588 (1971))

20. Rudakov, A., Shafarevich, I.: Inseparable morphisms of algebraic surfaces. Izvestia AN SSSR, Ser. Math. 40, 1264-1307 (1976); (Engl. Transl.: Math. USSR-Izvestia 10, 120-164 (1976))

21. Rudakov, A., Shafarevich, I.: K3-surfaces over fields of positive characteristic, Modern Problems in Mathematics, t. 18, Moscow, VINITT, 1981 (Engl. Transl.: J. Soviet. Math. 22, 1476-1533 (1983))

22. Shioda, T., Inose, H.: On singular K3 surfaces, in Complex Analysis and Algebraic Geometry, in honor of K. Kodaira. Cambridge Univ. Press. 1977, pp. 119-136

23. Verra, A.: The etale double covering of an Enriques' surface (preprint)

24. Vinberg, E.: Some discrete groups in Lobacevskii spaces. In: Discrete subgroups of Lie groups. Oxford University Press, 1975, pp. 323-348

25. Ueno, K.: A remark on automorphisms of Enriques surfaces. J. Fac. Sci. Univ. of Tokyo, Sec. IA 23, 149-165 (1976)

Oblatum 4-III-1983

\section{Note added in proof}

As was shown recently by Y. Namikawa, a better version of Horikawa's Global Torelli Theorem for Enriques surfaces gives another proof of the main result of this paper. 